\title{
Coronary artery calcium scanning: a useful tool for refining heart failure risk prediction?
}
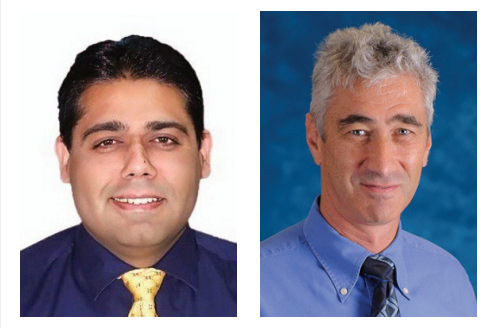

\author{
"While coronary artery calcium has been \\ used extensively to predict future \\ cardiovascular disease events, recent data \\ suggest it may be useful for identifying \\ congestive heart failure in early stages, \\ especially in asymptomatic populations."
}

\section{Muhammad A Latif ${ }^{1}$ \& Matthew J Budoff*1}

'Los Angeles Biomedical Research Institute, UCLA Harbor Medical Center, 1124 W Carson Street.

Torrance, CA 90502, USA

*Author for correspondence: Tel. : +1 3102224107 = Fax: +1 3107829652 = mbudoff@labiomed.org

Heart failure (HF) is a leading cause of morbidity and mortality in the elderly population, both in the developing and the developed nations. Approximately 5.8 million people in the USA currently have HF [1]. About one in five people who have HF die within 1 year after diagnosis as most of them when diagnosed are already at an advanced stage of the disease $[1,2]$. Unfortunately, physical examination only correctly diagnoses half of the cases. Early diagnosis and treatment of HF can halt the progression of disease and result in better life expectancy. There are some hurdles in accurately diagnosing HF in certain population groups. Diagnosing obese or elderly patients with congestive HF (CHF) can be challenging, especially given comorbidities, use of multiple medications and access to diagnostic services. While coronary artery calcium (CAC) has been used extensively to predict future cardiovascular disease (CVD) events, recent data suggest it may be useful for identifying CHF in early stages, especially in asymptomatic populations.

\section{Background of CAC as a tool for risk prediction in the asymptomatic population}

CAC has proved to be a great tool to diagnose and predict future outcome of various CVDs [1-3]. The accuracy of cardiac computed tomography (CT) has allowed identification of early atherosclerotic disease in asymptomatic individuals and prognostically surpassed traditional risk factors for the prediction of coronary artery disease (CAD). CAC as a risk stratification tool has great implications in diagnosing preclinical atherosclerosis [1]. In asymptomatic patients, the absence of CAC predicts excellent event-free survival with 10 -year event rates of approximately $1 \%$ [2]. Traditional risk factor evaluation has been shown to underrate coronary risk in certain population groups, particularly in women and young adults [3,4]. This may lead to suboptimal medical management of their coronary risk factors. CAC may help to refine the risk stratification and treatment strategies of these patient populations who are otherwise categorized as intermediate or low risk based on Framingham Risk Score categorization. CAC has consistently demonstrated independent and incremental predictive power for CVD when added to traditional risk factors [5-11].

In the Multi-Ethnic Study of Atherosclerosis (MESA) cohort [12], many traditional cardiovascular risk factors were associated with both the risk of developing cardiovascular events and increases in existing CAC scores. These included older age, male gender, white race/ethnicity, hypertension, higher BMI, diabetes mellitus and a family history of heart attack. Diabetes mellitus was the strongest risk factor for CAC progression. CAC progression is associated with mortality even after adjusting for baseline CAC, age, sex, time between scans, hypertension, hypercholesterolemia, diabetes, family history and smoking. Despite excellent cost-effectiveness data and prognostication, concerns have been raised about radiation. With modern CT use, the radiation exposure associated with CAC scans has been significantly reduced, now comparable to one to two mammograms ( 1 mSv) [13-15], while providing important information about the coronary risk and cardiac anatomy of the subject. Patient-specific protocols, limited scan length and lowering tube voltage further reduce the radiation dose to the individual patient.

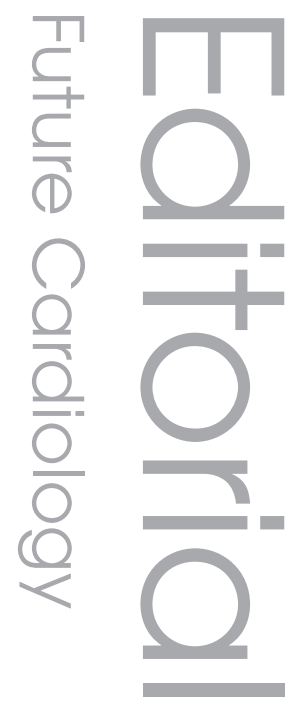

\section{Keywords}

- computed tomography - coronary artery calcification $=\mathrm{HF}=$ risk prediction of $\mathrm{HF}$

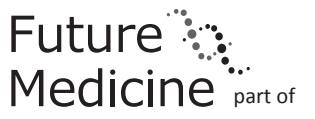


Use of CAC for diagnosis of CHF

The etiology of HF, whether coronary induced or nonischemic, may be difficult to discern clinically. Differentiation of ischemic from nonischemic etiology is clinically important for both therapeutic and prognostic implications. With its ability to noninvasively discern and quantitate coronary artery calcification, studies originally performed with electron beam tomography correlated well with angiographic stenosis and were shown to be useful in distinguishing ischemic from nonischemic cardiomyopathies [16]. The current standard reference to differentiate between ischemic and nonischemic cardiomyopathy $(\mathrm{CM})$ is invasive angiography. A study was carried out to compare the sensitivity and specificity of CAC testing with angiography in the setting of CHF of unclear etiology. One hundred and twenty-five patients with CM (ejection fraction $<0.40$ ) and known coronary anatomy underwent CAC scanning. Of the 72 patients who were found to have ischemic CM, 71 patients had CAC (sensitivity 99\%; $\mathrm{p}<0.001$; mean score $798 \pm 899$ ). In comparison, among the 53 patients without significant coronary artery disease (nonischemic CM), the mean score was significantly lower $(17 \pm 51$; $\mathrm{p}<0.0001)$, and 44 patients had a CAC score of 0 (no CAC present). Overall accuracy for determining the etiology of CM (differentiating ischemic from nonischemic) was $92 \%$ for this technique.

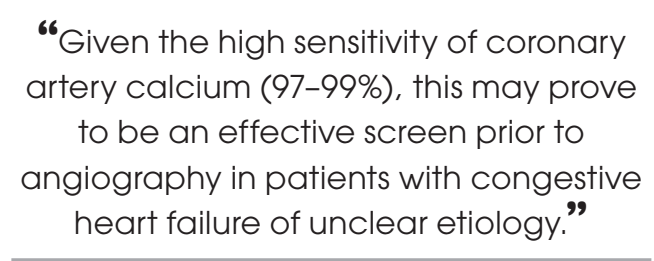

Determining the etiology of the $\mathrm{CM}$ in a patient presenting with HF can be difficult. Both dilated and ischemic CM may have identical clinical presentations with cardiomegaly, reduced left ventricular function and CHF. The treatment and prognosis of these two diseases aredifferent, and therefore diagnosis is critical for optimal management. Noninvasive studies have shown that perfusion defects could distinguish between ischemic and nonischemic CM with only fair accuracy, and that perfusion defects can occur in idiopathic dilated CM as well as in ischemic CM. Nuclear imaging may have artifacts and attenuation variations that can lead to incorrect diagnoses, and the common presence of left bundle branch block in CM, which can also make interpretation of nuclear imaging difficult. CAC testing was compared with nuclear testing in this population, and the overall diagnostic accuracy was significantly higher with CT, as compared with nuclear stress testing (84 vs $64 \% ; p=0.009)$. Given the high sensitivity of CAC (97-99\%), this may prove to be an effective screen prior to angiography in patients with CHF of unclear etiology [17]. In another study, the accuracy of CAC was compared with that of echocardiography in 111 patients undergoing angiography for the evaluation of HF. Electron beam tomography-derived coronary calcium correctly identified 61 out of 63 patients $(97 \%)$ with obstructive coronary artery disease, significantly higher than the 43 out of 63 patients $(68 \%)$ with segmental wall motion abnormalities identified by echocardiography $(\mathrm{p}<0.001)[18]$. The authors found that the presence of CAC is superior to that of segmental wall motion abnormalities by echocardiography to distinguish ischemic from nonischemic CM.

\section{Use of CAC in risk prediction of CHF}

With advances in CVD treatment, more people now survive heart attacks but are left with a weakened heart. Effective treatment of HF not only reduces the mortality but also slows the progression of the disease. Newer prognostic studies in asymptomatic cohorts have started to demonstrate the ability of CAC testing to identify those individuals who develop CHF. Recently, $\mathrm{CAC}$ has been shown to have a clear association with the risk of HF [19]. In the Rotterdam Study, a population-based cohort, 1897 asymptomatic participants (mean age 69.9 years; $58 \%$ women) underwent $\mathrm{CAC}$ scoring and were followed for 6.8 years for the occurrence of HF and CHD. After adjustment for cardiovascular risk factors, increasing CAC scores were associated with HF ( $\mathrm{p}$ for trend $<0.001$ ), with a hazard ratio of 4.1 (95\% CI: 1.7-10.1) for CAC scores $>400$ compared with CAC scores of $0-10$. Moreover, adding CAC to cardiovascular risk factors substantially improved the risk classification of subjects (continuous net reclassification index: $34.0 \%$ ). Because HF is highly prevalent in the elderly, it might be valuable to include $\mathrm{HF}$ as an outcome in future risk assessment programs incorporating CAC. One hypothesis is that $\mathrm{CAC}$ may predispose to $\mathrm{CHF}$, as a marker of endothelial function, independent of overt CAD. Alternatively, those with more CAC may just have more cardiovascular events and, thus, if they survive, will ultimately develop CHF as a sequelae of multiple heart attacks (CAC as 
a marker of ischemic CM). The Health $\mathrm{ABC}$ HF model adequately predicted 5-year HF risk in a large community-based study, providing support for the external validity of the model. This tool may be used to identify individuals in whom to target HF prevention efforts [20]. The Health ABC HF prediction model uses common clinical variables to predict HF incident risk in the elderly, an approach that may be used to target and treat high-risk individuals, and consideration should be made to add CAC to this model to make it more robust in identifying preclinical cases of HF. Further long-term outcome studies in the elderly will need to be performed to validate the current observations, but the robust net reclassification offered by $\mathrm{CAC}$ is appealing.

\section{Financial \& competing interests disclosure \\ The authors have no relevant affiliations or financial involvement with any organization or entity with a financial interest in or financial conflict with the sub- ject matter or materials discussed in the manuscript. This includes employment, consultancies, honoraria, stock ownership or options, expert testimony, grants or patents received or pending, or royalties. \\ No writing assistance was utilized in the production of this manuscript.}

\section{References}

1. Budoff MJ. Screening for ischemic heart disease with cardiac CT: current recommendations: scientific. Scientifica 8 , 1-12 (2012).

2. Blaha M, Budoff MJ, Shaw LJ et al. Absence of coronary artery calcification and all-cause mortality. JACC Cardiovasc. Imaging 2(6), 692-700 (2009).

3. Lakoski SG, Greenland P, Wong ND et al. Coronary artery calcium scores and risk for cardiovascular events in women classified as 'low risk' based on Framingham risk score: the multi-ethnic study of atherosclerosis (MESA). Arch. Intern. Med. 167(22), 2437-2442 (2007).

4. Ford ES, Giles WH, Mokdad AH. The distribution of 10-year risk for coronary heart disease among US adults: findings from the National Health and Nutrition Examination Survey III. J. Am. Coll. Cardiol. 43, 1791-1796 (2004).

5. Arad Y, Goodman KJ, Roth M, Newstein D, Guerci AD. Coronary calcification, coronary disease risk factors, C-reactive protein, and atherosclerotic cardiovascular disease events: the St. Francis Heart Study. J. Am. Coll. Cardiol. 46(1), 158-165 (2005).

6. Detrano R, Guerci AD, Carr JJ et al. Coronary calcium as a predictor of coronary events in four racial or ethnic groups. N. Engl. J. Med. 358, 1336-1345 (2008).

7. Budoff MJ, Shaw LJ, Liu ST et al. Long-term prognosis associated with coronary calcification: observations from a registry of 25,253 patients. J. Am. Coll. Cardiol. 49(18), 1860-1870 (2007).

8. Greenland P, LaBree L, Azen SP, Doherty TM, Detrano RC. Coronary artery calcium score combined with Framingham score for risk prediction in asymptomatic individuals. JAMA 291(2), 210-215 (2004).

9. Becker A, Leber A, Becker C, Knez A. Predictive value of coronary calcifications for future cardiac events in asymptomatic individuals. Am. Heart J. 155, 154-160 (2008).

10. Nasir N, Shaw LJ, Liu ST et al. Ethnic differences in the prognostic value of coronary artery calcification for all-cause mortality. J. Am. Coll. Cardiol. 50, 953-960 (2007).

11. Erbel R, Mohlenkamp S, Moebus S et al. Coronary risk stratification, discrimination, and reclassification improvement based on quantification of subclinical coronary atherosclerosis: the Heinz Nixdorf Recall study. J. Am. Coll. Cardiol. 56, 1397-1406 (2010).

12. Kronmal RA, McClelland RL, Detrano R et al. Risk factors for the progression of coronary artery calcification in asymptomatic subjects results from the Multi-Ethnic Study of Atherosclerosis (MESA). Circulation 115, 2722-2730 (2007).

13. Ficaro EP, Zanzonico P, Stabin MG et al. Variability in radiation dose estimates from nuclear and computed tomography diagnostic imaging. J. Nucl. Cardiol. 16(1), 161 (2009).

14. Budoff MJ, Gupta M. Radiation exposure from cardiac imaging procedures do the risks outweigh the benefits? J. Am. Coll. Cardiol. 56(9), 712-714 (2010).

15. Voros S, Rivera JJ, Berman DS et al. Guideline for minimizing radiation exposure during acquisition of coronary artery calcium scans with the use of multidetector computed tomography. A report by the Society for Atherosclerosis Imaging and Prevention Tomographic Imaging and Prevention
Councils in collaboration with the Society of Cardiovascular Computed Tomography. J. Cardiovasc. Comput. Tomogr. 5(2), 75-83 (2011).

16. Budoff MJ, Shavelle DM, Lamont DH et al. Usefulness of electron beam computed tomography scanning for distinguishing ischemic from nonischemic cardiomyopathy. JACC 32, 1173-1178 (1998).

17. Budoff MJ, Jacob B, Rasouli ML, Yu D, Chang RS, Shavelle DM. Comparison of electron beam computed tomography and technetium stress testing in differentiating cause of dilated versus ischemic cardiomyopathy. J. Comput. Assist. Tomogr. 29(5), 699-703 (2005).

18. Le T, Ko JY, Kim HT, Akinwale P, Budoff MJ. Comparison of echocardiography and electron beam tomography in differentiating the etiology of heart failure. Clin. Cardiol. 23(6), 417-420 (2000).

19. Leening MJ, Elias-Smale SE, Kavousi M et al. Coronary calcification and the risk of heart failure in the elderly: the Rotterdam study. Source Department of Epidemiology, Erasmus Medical Center, Rotterdam, the Netherlands; Department of Cardiology, Erasmus Medical Center, Rotterdam, the Netherlands. JACC Cardiovasc. Imaging 5(9), 874-880 (2012).

20. Kalogeropoulos A, Psaty BM, Vasan RS et al. Validation of the health $\mathrm{ABC}$ heart failure model for incident heart failure risk prediction: the Cardiovascular Health Study. Cardiovascular Health Study. Source Department of Medicine, Division of Cardiology, Emory University, Atlanta, GA 30322, USA. Circ. Heart Fail. 3(4), 495-502 (2010). 